\title{
PARALLEL PLATE WAVEGUIDE EXCITATION BY AN ARRAY OF LUMPED SOURCES
}

\author{
S. E. Bankov \\ Kotelnikov Institute of Radioengineering and Electronics of Russian Academy of Sciences, \\ Mokhovaya 11-7, Moscow 125009, Russia
}

The paper is received on July 23, 2019

\begin{abstract}
Boundary problem of parallel plate waveguide (PPW) excitation by an infinite array of metal strips with lumped sources is considered. The array operates in quasi-periodical regime. An effective analytical-numerical boundary problem solution and numerical algorithm on its base are proposed. Numerical results are discussed and compered with results obtained with help of High Frequency System Simulator (HFSS). Good agreement between HFSS numerical simulations and results of the proposed approach are demonstrated. It is shown that under some conditions array of lumped sources effectively excites PPW in ultra wide band (UWB) of operating frequencies.
\end{abstract}

Key words: parallel plate waveguide, lumped source, antenna array, quasi-periodical regime.

Аннотация. Решена задача о возбуждении плоского волновода бесконечной решеткой сосредоточенных источников. Решетка функционирует в квазипериодическом режиме. Предложено численно-аналитическое решение граничной задачи и эффективный численный алгоритм на его основе. Обсуждаются численные результаты и приводится их сравнение с результатами, полученными при помощи HFSS. Показано хорошее совпадение между данными полученными по приближенной модели и строгим решением граничной задачи. Показано, что при определенном выборе параметров структуры она может функционировать в сверхширокой полосе частот.

Ключевые слова. Плоский волновод, сосредоточенный источник, антенная решетка, квазипериодический режим. 


\section{Introduction}

PPW is a widely used for antenna design structure. A lot of leaky-wave antennas are constructed on the base of PPW [1-3]. We may also note PPW application in quasi optical planar multi-beam antennas [4-6]. A great advantage of PPW is its compliance to printed circuits technology. Usually PPW is a printed circuit board (PCB) and therefore such elements like for example radiating slots may be performed in frame of PCB manufacturing.

Last decades substrate integrated waveguides (SIW) and SIW devices became very popular $[7,8]$. They combine printed circuit low cost, waveguide low loss and are very perspective for millimeter wave applications. Such device is a combination of metallized holes in PPW and it may be simply combined with radiating structure in form of slotted array.

SIW technology may be successfully applied for planar multi-beam antenna design [4-6]. Planar mirrors and multi-channel radiators may be performed as a system of metallized holes in PCB. Typically multi-channel radiator is an array of metal cylinders connected from one side with PPW screen and from the other side with a transmission line (TRL) conductor. Thus such a cylinder is a PPW exciter that provides interaction between PPW waves and waves in TRL.

A typical way for PPW structures simulation is an application of systems of electromagnetic modeling like for example High Frequency System Simulator (HFSS) [9]. These systems have known advantages: flexibility, high precision etc. At the same time analytical or analytic-numerical models are relatively simple, do not require computer resources costs and may be simply physically interpreted. Thus development of such models is a useful step. This work is focused to development of an analytic-numerical model of PPW with array of exciters. We consider linear infinite array operating in a quasi-periodical regime.

\section{Boundary problem formulation}

The investigated structure is shown in fig. 1. It consists of a semi-infinite PPW and an infinite linear array of thin sheets. Structure located in the left part of fig. 1 may be called as a resonator. This resonator on the one hand prevents backward 
radiation and on the other hand plays a positive role increasing operating frequency range of the considered device.

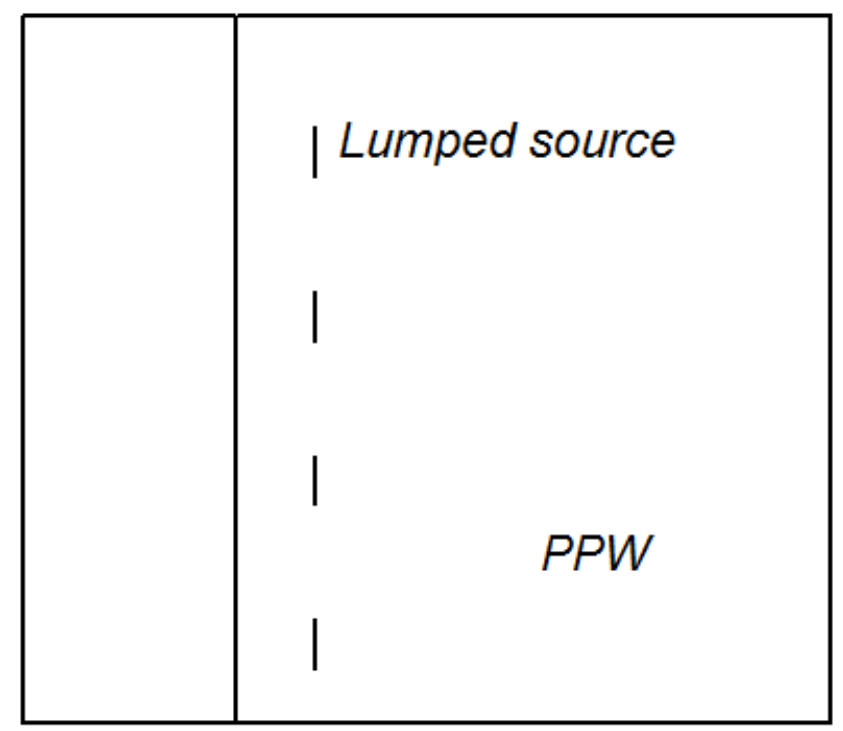

Resonator

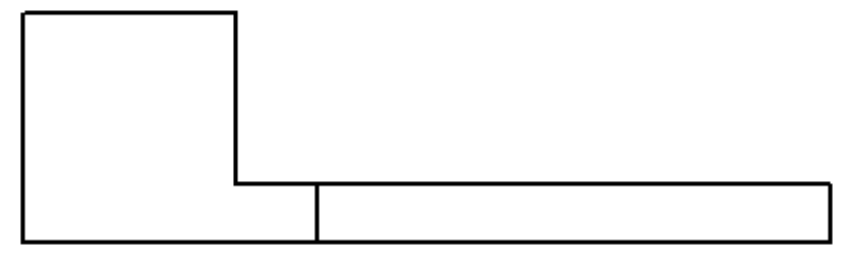

Fig. 1. Investigated structure.

Infinite array analysis in quasi-periodical regime may be reduced to the analysis of one period that is usually called as a Floquet channel [10]. It is shown in fig. 2.

Now we consider boundary conditions for electromagnetic field in this structure. Tangential electric field should be equal to zero on the perfect metal surfaces at $z=0, h, H, x=0,-L$. Boundary condition on the thin sheet is written as follows:

$$
U(y)=J(z) R_{g}+\mathrm{E},
$$

where $U$ is a voltage between conductors placed at $z=0, h$ :

$$
U=\int_{0}^{h} E_{z}(y, z) d z,
$$

$J$ is a current flowing along the sheet: 


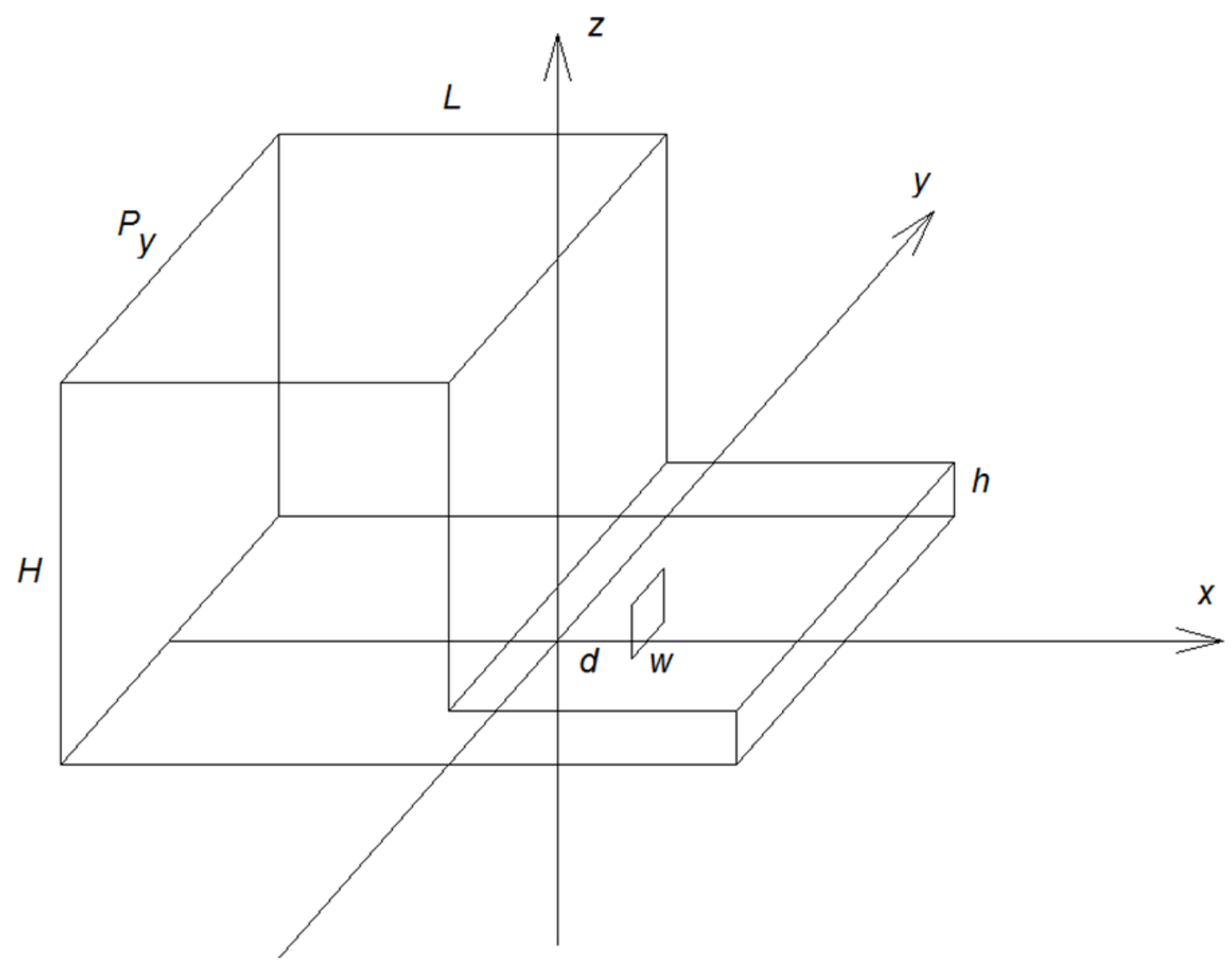

Fig. 2. Floquet channel.

$$
J(z)=\int_{-w / 2}^{w / 2} I_{z}(y, z) d y .
$$

Here $I_{z}(y, z)$ is electric current density. Parameters E, $R_{g}$ in relation (1) are electromotive force and inner impedance of the lumped source associated with the given sheet. Expression (1) is an analog of Ohm's law. It is written under several assumptions. We suppose that parameters $w, h$ satisfy inequalities:

$$
k h, k w<<1,
$$

where $k$ is a free space wavenumber. We may assume that electric current density and electric field do not vary along $0 z$ axis if conditions (4) are satisfied. In this case relation (1) may be written in a new form:

$$
h E_{z}(y)=J R_{g}+\mathrm{E} .
$$

Dependence on coordinate $y$ is still presented in expression (5). We may average formula (5) on interval $|y|<w / 2$ to eliminate this dependence: 


$$
\frac{h}{w} \int_{-w / 2}^{w / 2} E_{z}(y) d y=J R_{g}+\mathrm{E} .
$$

Lumped source which parameters are presented in (6) allows one to describe PPW excitation. This source performs function of a Floquet channel port. Let it has number 1 . Waveguide located in the right part of fig. 2 forms port 2. It has height $h$ and width $P_{y}$. Thus the Floquet channel is a two port junction that connects lumped source and waveguide. Typically lumped port serves as a model of transmission line. Therefore one may conclude that the considered structure is a transition of some kind. It connects transmission line and waveguide.

Fields on surfaces at $y= \pm P_{y} / 2$ satisfy boundary conditions of periodicity:

$$
E\left(-P_{y} / 2\right) \exp \left(-i \kappa_{y} P_{y}\right)=E\left(P_{y} / 2\right),
$$

here $E\left( \pm P_{y} / 2\right)$ may be an arbitrary component of electromagnetic field, $\kappa_{y}$ - s a parameter of quasi-periodical regime. It determines phase shift between the array channels located along $0 y$ axis.

We should note that assumption about field independence on coordinate $z$ is valid not only on the sheet surface but in the whole region $x>0$. We should find electromagnetic field that satisfies Maxwell equations and boundary conditions formulated above.

\section{Boundary problem solution}

In order to solve formulated above problem we separate our complicated structure to several more simple regions and write fields in them in form satisfying Maxwell equations and a part of boundary conditions. We select as a partial regions 1-3 sections located at $-L<x<0,0<x<d$ and $x>d$.

Region 1 is a resonator, region 2 is a section of rectangular waveguide with length $d$ and transversal dimensions $h, P_{y}$ and region 3 is a semi-infinite waveguide with the same dimensions. A specificity of the waveguide is boundary conditions (7) specified on its vertical walls. 
With help of inequalities (4) we provide field independence on coordinate $z$. Let us also assume that component of electric field $E_{z}$ is much greater than components $E_{x, y}$. Such field presentation is typical for field in PPW with electrically small height $h$.

We express electromagnetic field in regions $1-3$ through components $E_{z}, H_{z}$ , so as a sum of electric and magnetic waves [11]. In frame of accepted assumptions one may demonstrate that electric waves with $H_{z}=0$ completely describe field in regions 2,3. Field in region 1 depends on coordinate $z$ and we should take into account contribution of magnetic waves. In accordance with above notes we have the following relations:

$$
\begin{aligned}
& E_{z 1}=\sum_{n, m} A_{n, m}^{e} \cos \alpha_{m} z \operatorname{sh} \gamma_{n, m}(x+L) \exp \left(-i \kappa_{n} y\right), \\
& H_{z 1}=\sum_{n, m} A_{n, m}^{h} \sin \alpha_{m} z \operatorname{ch} \gamma_{n, m}(x+L) \exp \left(-i \kappa_{n} y\right), \\
& E_{z 2}=\sum_{n}\left(B_{n} \operatorname{ch} \beta_{n} x+C_{n} \operatorname{sh} \beta_{n} x\right) \exp \left(-i \kappa_{n} y\right), \\
& E_{z 3}=\sum_{n} D_{n} \exp \left(-i \kappa_{n} y-\beta_{n}(x-d)\right), \\
& \kappa_{n}=\kappa_{y}+\frac{2 \pi n}{P_{y}}, \\
& \alpha_{m}=\frac{\pi m}{H}, \\
& \gamma_{n, m}=\sqrt{\kappa_{n}^{2}+\alpha_{m}^{2}-k^{2}}, \\
& \beta_{n}=\gamma_{n, 0}, \\
& n=\ldots-1,0,1, \ldots, \\
& m=0,1, \ldots \\
& m,
\end{aligned}
$$


Fields (9) satisfy Maxwell equations, boundary conditions on perfectly conducting surfaces and conditions of periodicity. We have to satisfy the rest boundary conditions on planes $x=0$ and $x=d$. Let us start with plane $x=0$.

Denote electric field at $x=0,0<z<h$ as $\mathrm{E}_{z}$. Function $\mathrm{E}_{z}$ is equal to zero at $z>h$ in accordance with boundary conditions on metal surface. Another tangential to plane $x=0$ component $\mathrm{E}_{y}$ is equal to zero at $x=0,0<z<H$ because of above boundary conditions and assumption about field character in regions 2,3 at $0<z<h$.

Next we express electric field component $E_{y}$ in region 1 through components $E_{z}, H_{z}$ with help of known relations [11] and equate it to zero at $x=0,0<z<H$. Taking into account orthogonality of functions $\sin \alpha_{m} z$ on interval $0<z<H$ and functions $\exp \left(-i \kappa_{n} y\right)$ on interval $|y|<P_{y} / 2$ we obtain as a result the following relation between coefficients $A_{n, m}^{e, h}$ :

$$
A_{n, m}^{h}=-\frac{\alpha_{m} \kappa_{n}}{k W_{0} \gamma_{n, m}} A_{n, m}^{e},
$$

where $W_{0}$ is a free space wave impedance.

Now let us express coefficients $A_{n, m}^{e}$ through electric field $\mathrm{E}_{z}$. Equating expression (9) for component $E_{z}$ at $x=0$ to function $\mathrm{E}_{z}$ and applying once more orthogonality of trigonometric and exponential functions we have:

$$
\begin{aligned}
& A_{n, m}^{e}=\frac{\varepsilon_{m}}{P_{y} H \operatorname{sh} \gamma_{n, m} L} \frac{\sin \alpha_{m} h}{\alpha_{m}} e_{n}, \\
& e_{n}=\int_{-P_{y} / 2}^{P_{y} / 2} \mathrm{E}_{z}(y) \exp \left(i \kappa_{n} y\right) d y, \\
& \varepsilon_{m}=\left\{\begin{array}{l}
1, m=0, \\
2, m>0 .
\end{array}\right.
\end{aligned}
$$


In accordance with boundary conditions at $x=0,0<z<h$ electric field in region 1 should be equal to electric field in region 2. Due to it we may find coefficients $B_{n}$, expressing them through field $\mathrm{E}_{z}$ :

$$
B_{n}=\frac{e_{n}}{P_{y}}
$$

Thus with help of relations (11) и (12) we satisfy boundary conditions for electric field at $x=0$. Boundary conditions for magnetic field require continuity of components $H_{z, y}$ at $x=0,0<z<h$. It is impossible to satisfy this requirement completely because we assume that $\mathrm{E}_{y}=0$. Because of it we may provide continuity only for component $H_{y}$.

Component $H_{y}$ in regions 1,2 may be written with help of formula (10) and relations from [11] in form of expansions (9):

$$
\begin{aligned}
& H_{y 1}=\sum_{n, m}-\frac{i \cos \alpha_{m} z \operatorname{ch} \gamma_{n, m}(x+L) \exp \left(-i \kappa_{n} y\right)}{k W_{0} \gamma_{n, m}}\left(\kappa_{n}^{2}-k^{2}\right) A_{n, m}, \\
& H_{y 2}=\sum_{n}-\frac{i \beta_{n} \exp \left(-i \kappa_{n} y\right)}{k W_{0}}\left(B_{n} \operatorname{sh} \beta_{n} x+C_{n} \operatorname{ch} \beta_{n} x\right) .
\end{aligned}
$$

Here and below we exclude upper index $e$ in coefficients $A_{n, m}^{e}$. Let us introduce designation:

$$
Q_{n}=\frac{\left(\kappa_{n}^{2}-k^{2}\right)}{k^{2} P_{y} H h} \sum_{m} \frac{\varepsilon_{m}}{\gamma_{n, m}} \operatorname{cth} \gamma_{n, m} L\left(\frac{\sin \alpha_{m} h}{\alpha_{m}}\right)^{2} .
$$

To satisfy boundary conditions at $x=0,0<z<h$ we equate fields (13) at $x=0$. Next we integrate the obtained relation over $z$ and apply functions $\exp \left(-i \kappa_{n} y\right)$ orthogonality. As a result we find coefficients $C_{n}$ :

$$
C_{n}=\frac{k^{2} Q_{n}}{\beta_{n}} e_{n}
$$


At the next step of the solution we satisfy boundary conditions at $x=d$. As it is following from chapter 3 electric current with density $I_{z}$ flows $x=d, 0<z<h$, $|y|<w / 2$. Tangential electric field is continuous on a sheet of electric current and magnetic field has a discontinuity equal to current density [11]. Hence we obtain the following relations:

$$
\begin{aligned}
& B_{n} \operatorname{ch} \beta_{n} d+C_{n} \operatorname{sh} \beta_{n} d=D_{n}, \\
& D_{n}+B_{n} \operatorname{sh} \beta_{n} d+C_{n} \operatorname{ch} \beta_{n} d=\frac{j_{n} k W_{0}}{i \beta_{n} P_{y}}, \\
& j_{n}=\int_{-w / 2}^{w / 2} I_{z}(y) \exp \left(i \kappa_{n} y\right) d y .
\end{aligned}
$$

Substituting expressions (12), (15) to (16) we obtain:

$$
\begin{aligned}
& \frac{e_{n}}{P_{y}} \operatorname{ch} \beta_{n} d+\frac{k^{2} Q_{n}}{\beta_{n}} e_{n} \operatorname{sh} \beta_{n} d=D_{n}, \\
& D_{n}+\frac{e_{n}}{P_{y}} \operatorname{sh} \beta_{n} d+\frac{k^{2} Q_{n}}{\beta_{n}} e_{n} \operatorname{ch} \beta_{n} d=\frac{j_{n} k W_{0}}{i \beta_{n} P_{y}} .
\end{aligned}
$$

Next we express with help of system (17) coefficients $e_{n}$ through $j_{n}$ :

$$
e_{n}=\frac{k W_{0} \exp \left(-\beta_{n} d\right)}{i \beta_{n}\left(1+\frac{Q_{n} k^{2} P_{y}}{\beta_{n}}\right)} j_{n} \text {. }
$$

Now we may find electric field $E_{z}$ at $x=d$ and begin to satisfy boundary condition (6):

$$
E_{z}=\sum_{n} \frac{k W_{0}\left(\operatorname{ch} \beta_{n} d+\operatorname{sh} \beta_{n} d \frac{Q_{n} k^{2} P_{y}}{\beta_{n}}\right) \exp \left(-\beta_{n} d-i \kappa_{n} y\right) j_{n}}{i \beta_{n} P_{y}\left(1+\frac{Q_{n} k^{2} P_{y}}{\beta_{n}}\right)} .
$$

We approximate current on a narrow strip by a constant: 


$$
I_{z}(y)=C \text {. }
$$

Substituting relation (19) to equation (6) we find the constant $C$ :

$$
\begin{aligned}
& C=\frac{\mathrm{E}}{\frac{k h W_{0}}{2 i w P_{y}} \sum_{n}\left(1+\frac{\beta_{n}-Q_{n} k^{2} P_{y}}{\beta_{n}+Q_{n} k^{2} P_{y}} \exp \left(-2 \beta_{n} d\right)\right) \frac{f_{n}^{2}}{\beta_{n}}-w R_{g}}, \\
& f_{n}=2 \frac{\sin \kappa_{n} w / 2}{\kappa_{n}} .
\end{aligned}
$$

Now it is easy to find current $J$ and port 1 input impedance $Z_{i 1}$ :

$$
Z_{i 1}=\frac{i k h W_{0}}{2 w^{2} P_{y}} \sum_{n}\left(1+\frac{\beta_{n}-Q_{n} k^{2} P_{y}}{\beta_{n}+Q_{n} k^{2} P_{y}} \exp \left(-2 \beta_{n} d\right)\right) \frac{f_{n}^{2}}{\beta_{n}} .
$$

Reflection coefficient from port $1 S_{11}$ is simply related with impedance $Z_{i 1}$ :

$$
S_{11}=\frac{Z_{i 1}-R_{g}}{Z_{i 1}+R_{g}}
$$

The next interesting for us scattering parameter is transmission coefficient from port 1 to port $2 S_{21}$. We have to find wave amplitude in waveguide at $x>d$ when port 1 is excited. It is easy to do applying relations (9), (17), (18). With help of them we obtain the following expression for electric field amplitude of a transmitted wave $E_{z t}$ :

$$
E_{z t}=-\frac{i C k W_{0} f_{0}}{2 P_{y} \beta_{0}}\left(1+\frac{\beta_{0}-Q_{0} k^{2} P_{y}}{\beta_{0}+Q_{0} k^{2} P_{y}} \exp \left(-2 \beta_{0} d\right)\right) .
$$

Here constant $C$ is defined by formula (21). Transmitted wave power $P_{t}$ is simply related with electric field amplitude (24):

$$
P_{t}=-\frac{i \beta_{0}\left|E_{z t}\right|^{2} h P_{y}}{2 k W_{0}} .
$$

Power of incident on port 1 wave $P_{i}$ may be expressed through lumped source parameters: 


$$
P_{i}=\frac{|\mathrm{E}|^{2}}{8 R_{g}} .
$$

Then in accordance with S-matrix definition [12] we have:

$$
S_{21}=\sqrt{\frac{P_{t}}{P_{i}}} \exp \left(i \arg E_{z t}\right) .
$$

The rest two elements of scattering matrix $S_{12,22}$ may be found from properties of a reciprocal and non-dissipative two port junction [12]:

$$
\begin{aligned}
& S_{12}=S_{21}, \\
& \left|S_{22}\right|=\left|S_{11}\right|, \\
& \arg S_{22}=-\arg S_{11}+2 \arg S_{12}+\pi .
\end{aligned}
$$

Thus the Floquet channel $S$-matrix is completely determined.

\section{Numerical results}

In this section we discuss results of numerical simulation. Frequency dependence of the transition reflection coefficient $S_{11}$ is shown in fig. 3-6. Curves 1-4 in fig. 3 are obtained for $L=13.5, H=14, d=0.5, P_{y}=15, h=1, w=2.5,3.5,4.5,5.5$. All dimensions here and below are presented in millimeters. Numerical results presented in fig. 3-6 are obtained for in-phase array excitation $\kappa_{y}=0$. It is seen that width $w$ sufficiently affects to reflection coefficient level in the central part of operating frequency range. Lower edge of the range almost does not depend on width $w$. Upper edge of the range depends on parameter $w$. It monotonically increases with $w$ growth.

For unambiguous definition of the operating frequency range we have to specify maximum level of the reflection coefficient. Let it be equal to - 10 дБ. In this case as it is following from fig. 4 operating frequency range $D$ is equal to 25 . We define parameter $D$ as relation between maximum and minimum frequencies of the operating frequency range. Thus we see that for given parameters PPW may effectively excited by the array of lumped sources in an ultra-wide frequency band.

Curves $1-4$ in fig. 4 demonstrate influence of distance $d$ to reflection coefficient frequency dependence. They are calculated for $L=13.5, H=14$, 
$d=0,0.5,1,1.5, P_{y}=15, h=1, w=5.5$. Curves behavior is similar to one shown in fig. 4. It is seen that lower frequency of the operating frequency range weakly depends on thin sheet position and on the contrary upper frequency depends on it significantly. Also reflection level in the central part of the range sufficiently depends on parameter $d$.

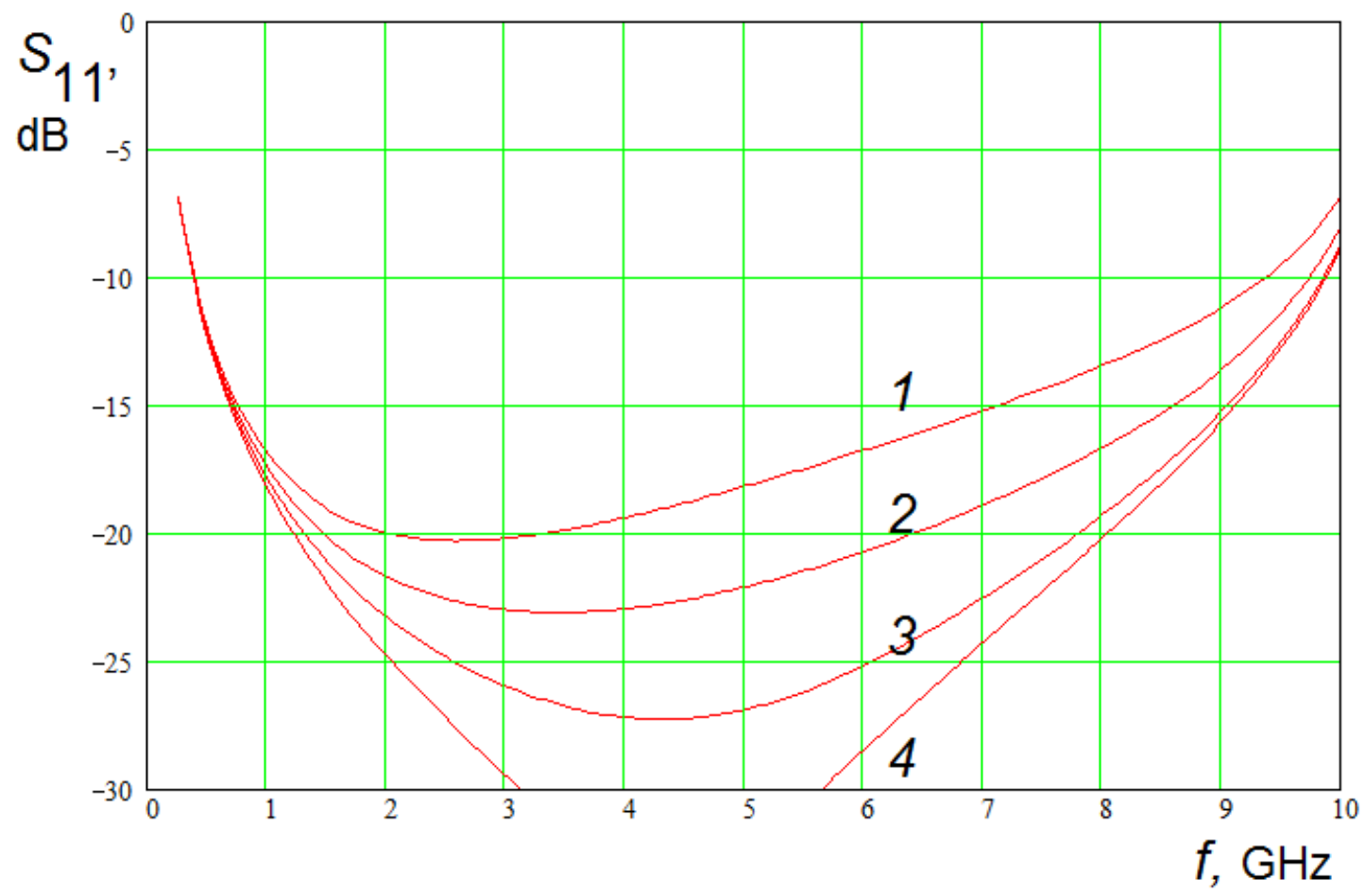

Fig. 3. Reflection coefficient frequency dependence for $w=2.5,3.5,4.5,5.5$.

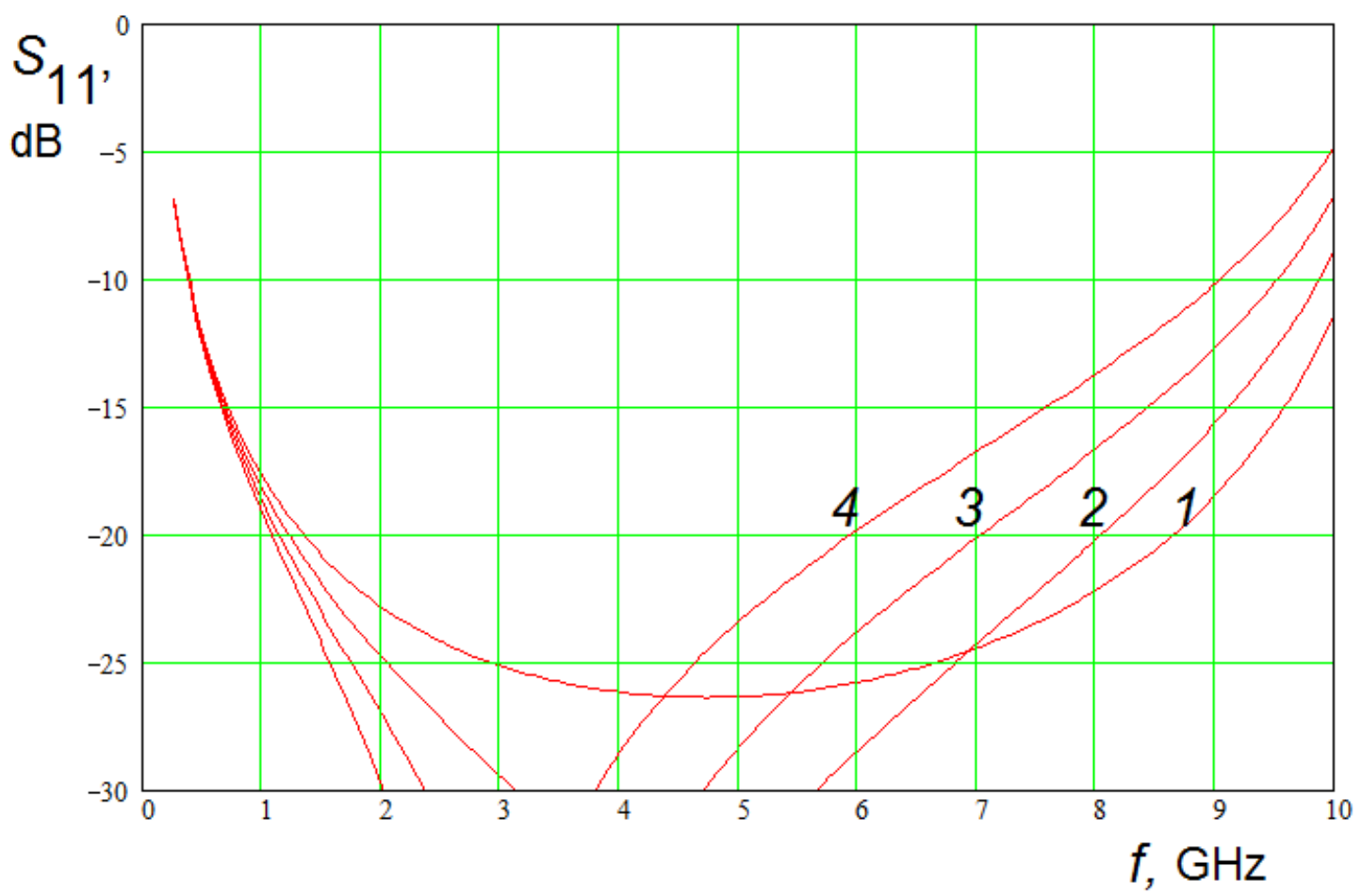

Fig. 4. Reflection coefficient frequency dependence for $d=0,0.5,1,1.5$. 


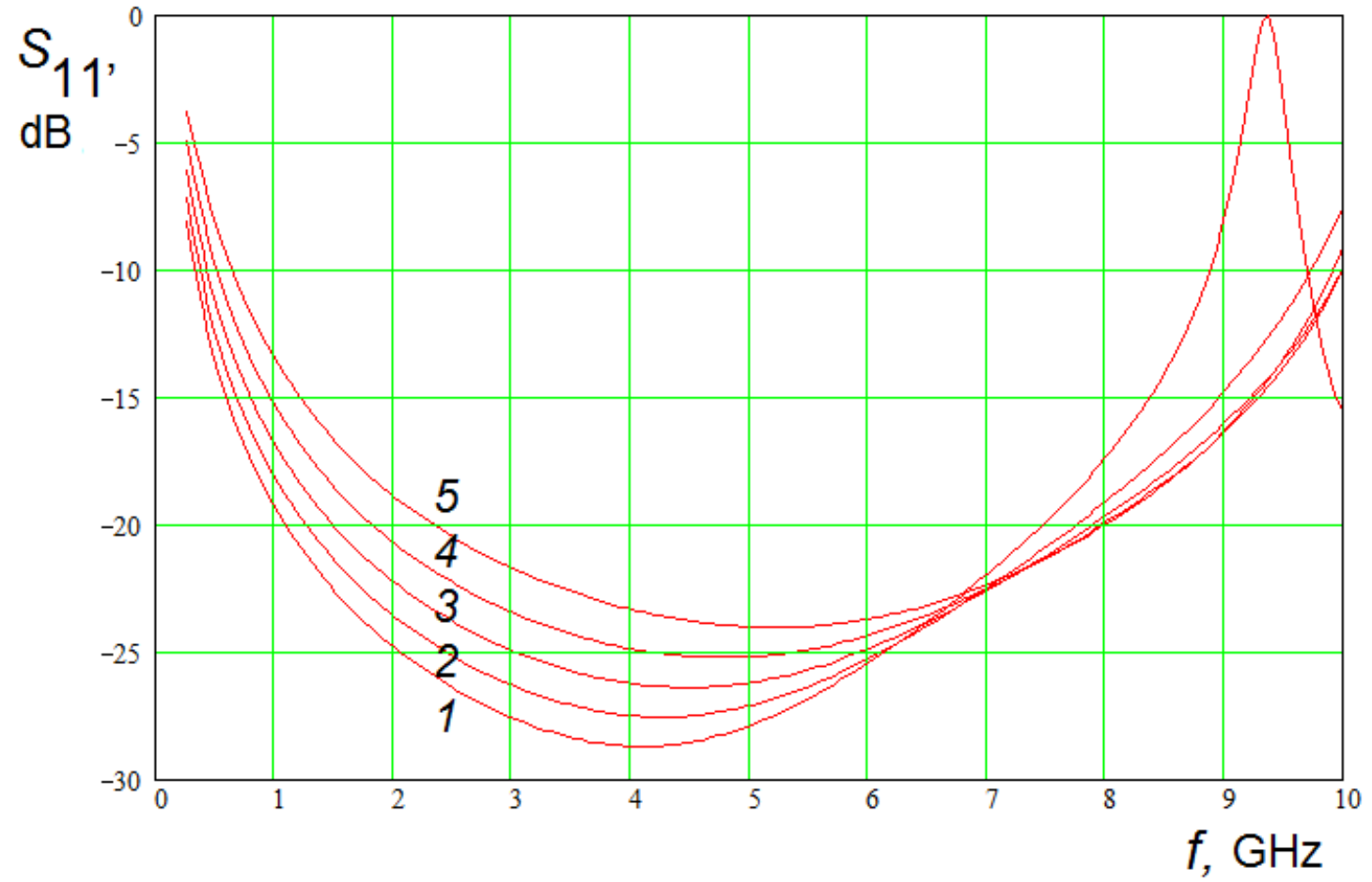

Fig. 5. Reflection coefficient frequency dependence for $L=16,14,12,10,8$.

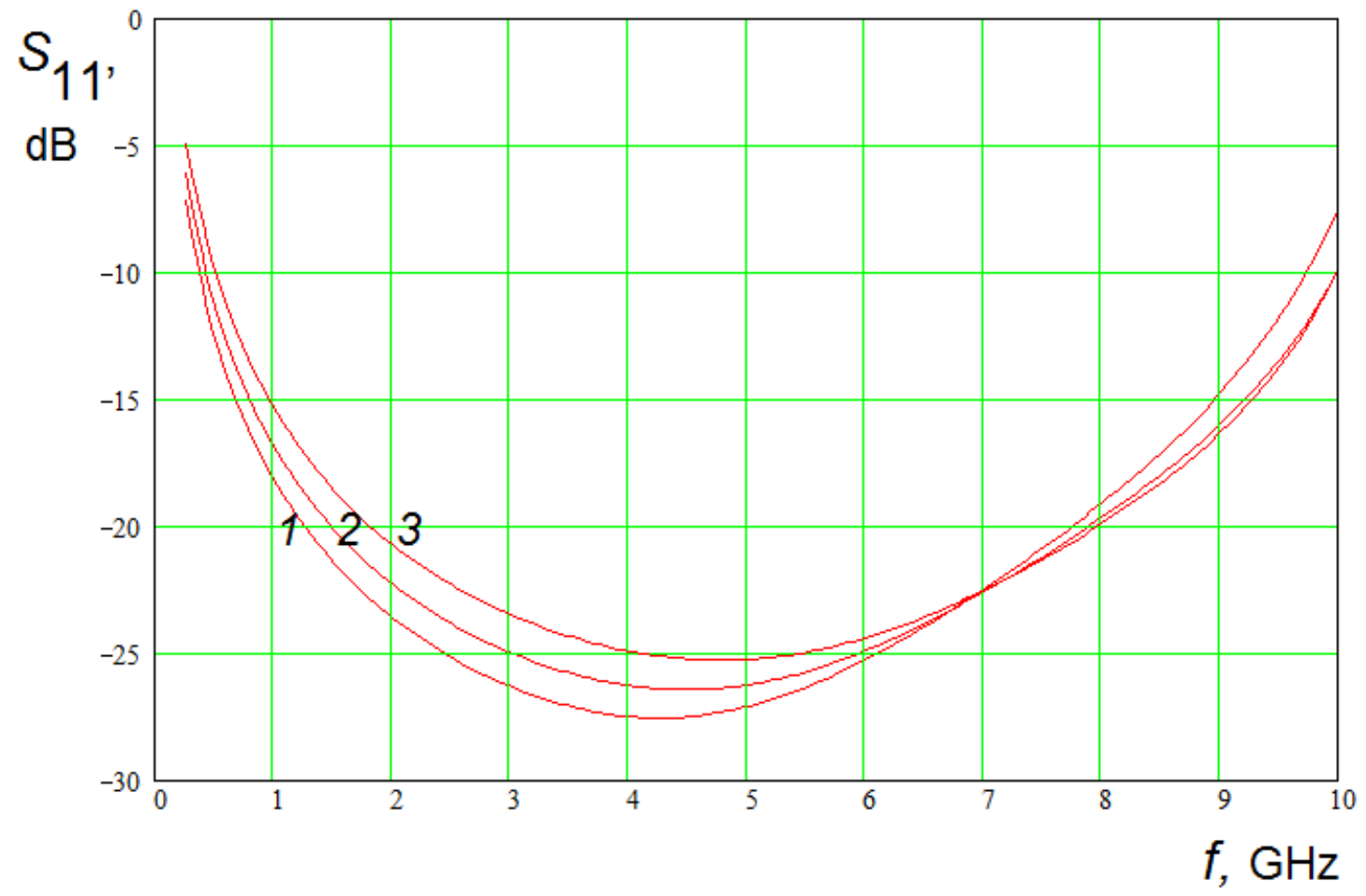

Fig. 6. Reflection coefficient frequency dependence for $H=10,12,14$.

Frequency dependence of reflection coefficient is shown in fig. 5. Curves $1-5$ correspond to $L=16,14,12,10,8, H=14, d=0.5, P_{y}=15, h=1, w=4.5$. They demonstrate influence of resonator $L$ on the device characteristics. It is seen that both lower and 
upper frequencies of the range depend on this parameter. The length increase decreases both frequencies.

The reflection coefficient behavior in dependence on resonator length demonstrates the main factors that determine operating frequency range of the device. The resonator input impedance has inductive character when $f \rightarrow 0$ and it causes mismatching of the array because inductive impedance tends to zero under these conditions.

We should note that inductivity value depends on the resonator dimensions: length $L$ and height $H$. It increases proportionally dimensions and thus lower frequency moves down.

On the other hand increase of the length $L$ influences on the device behavior at high frequencies. Here we understand under high frequencies such frequencies when the resonator dimensions are comparable with half wavelength. The dominant mode of the resonator is excited in this range. Input impedance of the resonator tends to zero at the resonance frequency and shunts lumped source. It causes reflection coefficient growth that determines upper operating frequency. Appearance of the resonance at high frequencies is well visible from curve 1 in fig. 5 .

Curves $1-3$ in fig. 6 are obtained for $L=14, H=10,12,14, d=0.5, P_{y}=15, h=1$, $w=4.5$. They demonstrate dependence of transition characteristics on height $H$. It is analogous to dependence on resonator length: increase of this parameter simultaneously decreases upper and lower edges of the operating frequency range.

For verification of the described above approximate transition model we analyzed the same structure with help of a standard system of electromagnetic simulation High Frequency System Simulator (HFSS). The transition model is shown in fig. 7. It has two ports: lumped and wave one located on the front surface of the model (see fig. 7).

Curves 1,2 in fig. 8 are obtained for $L=14, H=14, d=0.5, P_{y}=15, h=1, w=4.5$ with help of approximate model and finite elements method realized in HFSS respectively. One may see good agreement between two curves. Some difference between them is visible in the central part of frequency range. However reflection 
coefficient is small at these frequencies. Edges of the operating frequency range determined with help of approximate model are in a very good agreement with results of HFSS simulations.

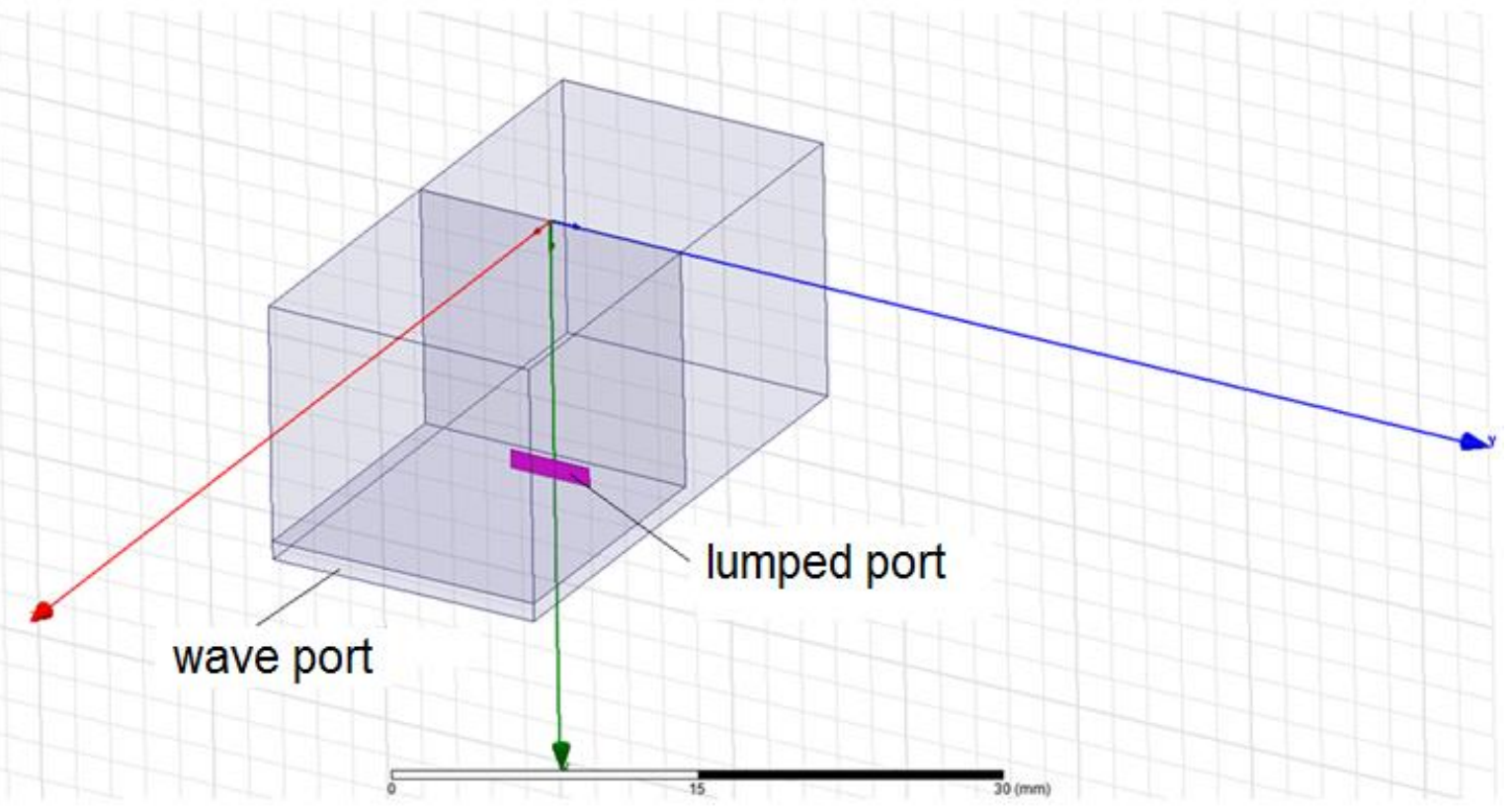

Fig. 7. Transition model.

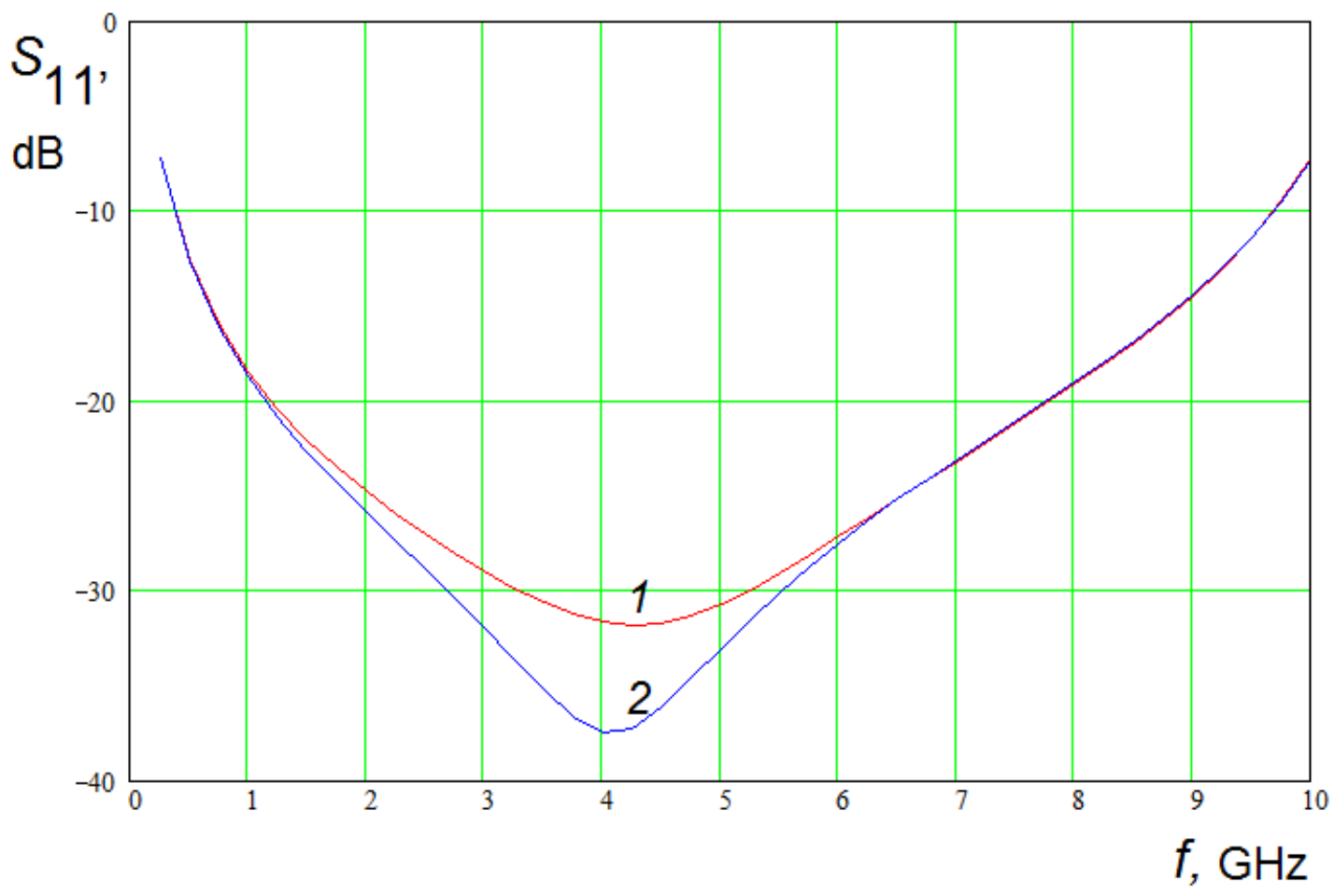

Fig. 8. Reflection coefficient frequency dependence: 1 - approximate model, $2-$ numerical simulation. 


\section{Conclusions}

Results presented above demonstrate high efficiency and good tolerance of the approximate analytic-numerical model. It may be used for fast analysis of PPW excitation by an array of lumped sources. Results presented above correspond to quasi-periodical regime. However this solution may be also applied for analysis of PPW excitation by one array element with help of solution integration over variable $\kappa_{y}$ in accordance with the known relations [13].

The work is performed in frame of state order (project 0030-2019-0014) and is supported by Russian Foundation of Base Researches (project No.18-07-00655 a).

\section{References}

1. Hirokawa J., Ando M., Goto N. Waveguide-Fed Parallel Plate Slot Array Antennas. IEEE Transaction on Antennas and Propagation. 1992. Vol.40. No. 2. P.218-223.

2. Hirokawa J., AndoM. Efficiency of $76 \mathrm{GHz}$ Post-Wall Waveguide-Fed Parallel Plate Slot Arrays. IEEE Transaction on Antennas and Propagation. 2000. Vol.48. No.11. P.1742-1745.

3. Kai H., Hirokawa J., Ando M. Circularly Polarized Post-Wall Waveguide Slotted Arrays. IEICE Electronics Express. 2004. Vol.1. No.1. P.7-12.

4. Ettorre M., Bruni S., Gerini G., Neto A., Llombart N., Maci S. Multi Sector PCSEBG Antenna for low cost high directivity application. IEEE AP-S Symposium, Honolulu, Hawaii, June 2007.

5. Albani M., Ettorre M., Maci S., Balling P., Gerini G., Pontoppidan K., Sipus Z., Sfoberg D., Vecchi G., Vipiana F. Reflector Surface Modeling. A European Collaboration, Eucap 2007, Edinburgh, UK, November 2007.

6. Ando M., Hirokawa J., Hirano T., Hyun P.S., Kai H. Advances in the design of single-layer slotted waveguide arrays and their system applications. ICECOM 2003, Dubrovnik, Croatia, P.352-359, October, 2003. 
7. Wu K., Deslandes D., Cassivi Y. The substrate integrated circuits -A new concept for high-frequency electronics and optoelectronics. Proc. 6-th Int. Conf. Telecomm. Modern Satellite, Cable and Broadcasting Service, Oct. 2003. Vol. 1. P. 3-5.

8. Sakakibara K. et. al. Single-layer Slotted Waveguide Arrays for Millimeter Wave Applications. IEICE Trans. Commun., 1996, Vol.E79-B, No.12, pp.1765-1772.

9. Bankov S.E., Kurushin A.A., Gutzait E.M. Resheniye opticheskikh $i$ mikrovolnovykh zadach s pomoshch'yu HFSS [Solution of optical and microwave problems with help of HFSS]. Moscow, Publishing house Orcada. 2012 (In Russian).

10. Bankov S.E., Kurushin A.A. Elektrodinamika dlya pol'zovateley SAPR [Electrodynamics for CAD users]. Moscow, Publishing house Solon-Press. 2018 (In Russian)

11. Markov G.T., Chaplin A.T. Vozbuzhdeniye elektromagnitnykh voln [Electromagnetic waves excitation]. Moscow, Radio i Svyaz' Publ., 1983 (In Russian)

12. Sazonov D.M. Mikrovolnovye ustroistva $i$ antenny [Microwave devices and antennas]. Moscow,Vysshaya Shkola Publ., 1988 (In Russian)

13. Hansen R. Scanning Array Antennas. New York, London. Academic Press. 1964.

\section{For citation:}

S. E. Bankov. Parallel plate waveguide excitation by an array of lumped sources Zhurnal Radioelektroniki - Journal of Radio Electronics. 2019. No.8. Available at http://jre.cplire.ru/jre/aug19/8/text.pdf

DOI 10.30898/1684-1719.2019.8. 